\title{
Linear and second-order nonlinear optical properties of non-fullerene acceptor derivatives with A-D-A structure
}

\author{
Lijing Gong ${ }^{1}$, Jing $\mathrm{Xu}^{2}$, Chunping $\mathrm{Li}^{1}$, Xiangyu Zhang ${ }^{1}$, and Zhi Jiang ${ }^{1}$ \\ ${ }^{1}$ Air Force Aviation University \\ ${ }^{2}$ Changchun Institute of Technology
}

September 24, 2021

\begin{abstract}
In this paper, in order to study the relationship between structure and performance, four new NFA derivatives were designed based on the two reported NFA molecules BO-4Cl and BTP-S2 by replacing the BT unit with a less-electron deficient BTz unit and inserting another ethylene double bond between the central core and the terminal groups. The DFT and TD-DFT calculations were applied to invstigate linear and nonlinear optical properties, such as electronic structure, electronic absorption, reorganization energy and the second-order NLO properties. The investigation demonstrates that they are all narrow bandgap derivatives, the absorption spectrum extends to the near-infrared region and using two ethylene double bond is the most effective way to reduce the energy gap, redshift the maximum absorption peak and the middle absorption band, enhance hole transport ability and weaken electron transport ability and enhance second-order NLO response. Considering the smaller electron and hole reorganization energy and the larger static first hyperpolarizability value, the studied NFA derivatives have great potential to become ambipolar charge transport materials and large second-order NLO materials.
\end{abstract}

\section{Hosted file}

paper.doc available at https://authorea.com/users/435167/articles/538373-linear-and-secondorder-nonlinear-optical-properties-of-non-fullerene-acceptor-derivatives-with-a-d-astructure 